\title{
Rapport aux savoirs des étudiantes moutahajibâte françaises
}

\author{
Amina Triki-Yamani
}

\begin{abstract}
Résumé
Cet article propose une synthèse des résultats principaux d'une recherche doctorale portant sur le rapport aux savoirs d'étudiantes françaises musulmanes, d'origine maghrébine, vivant en France, et dont la particularité est de porter le hijâb et de fréquenter régulièrement la mosquée. Dans un premier temps, en retraçant les stratégies identitaires de dix-sept étudiantes moutahajibâte, on a tenté de comprendre comment l'adoption de « la stratégie du retournement du stigmate » par ces jeunes femmes accentue leur rapport conflictuel aux savoirs laïques. Dans un second temps, on a exploré les fonctions psychiques du hijâb permettant de réajuster le « Moi-peau » chez ces jeunes femmes ainsi que leurs conflits internes des savoirs.
\end{abstract}

\begin{abstract}
This article presents a synthesis of a doctoral study relating to French Muslim women students' relation to knowledge. The participants are of North African origin; they live in France and their custom is to wear the hijâb and to attend the mosque regularly. First of all, the purpose of relating the records of seventeen veiled (moutahajibâte) students' identity strategies was to try to understand how adopting "the strategy of returning to stigmatization" by these young women increased their conflicting relationship to secular learning. Secondly, the psychic functions of the hijâb that permit the women to readjust the constancy of their self-identity of the young women as well as their internal learning conflicts was explored.
\end{abstract}

\section{PROBLÉMATIQUE ET MÉTHODOLOGIE}

Le choc qu'ont provoqué les affaires dites "du foulard islamique » à l'école française a rapidement été supplanté par une appréhension de l'islam de France, voire par l'engendrement d'une islamophobie. Il faut noter que l'islam revendiqué et affiché par une minorité musulmane estudiantine depuis le début des années 90 est un islam engagé et fondamentaliste ${ }^{6}$, très éloigné de l'islam traditionnel des parents et des grands-parents, importé du pays d'origine et pratiqué discrètement dans la sphère privée.

Plusieurs recherches universitaires en France (Gaspard, Khosrokhovar, 1995; Khosrokhovar, 1995-1996; Khosrokhovar, 1997; Venel, 1999; Weibel, 2000; Zouari, 2002) sont consacrées dès 1995 au foulard islamique en tant que

\footnotetext{
${ }^{6}$ M. S. AL-ASHMAWY distingue très nettement deux formes de fondamentalismes islamiques :

- un fondamentalisme "rationaliste" et spiritualiste qui, revenant à l'islam tel que le comprenaient les premiers musulmans, suit les enseignements du Coran et de la Sunna et les utilise pour renouveler les valeurs du travail, retrouver un rôle actif dans la civilisation mondiale, et enfin, définir précisément ce que doivent être les institutions politiques et juridiques de la société, sans sacralisation excessive et sans nihilisme destructeur, - un fondamentalisme activiste politique qui, loin de proposer un authentique renouvellement de la pensée religieuse, se bat uniquement sur le terrain et avec les armes de la politique, rabaissant l'islam et sa charîa (le droit musulman) au niveau d'une vulgate politique. (Al-Ashmawy, 1989, pp. 75-76.)
} 
donnée psychosociale dans la construction de la personnalité islamique de la jeune femme musulmane ${ }^{7}$. La plupart des chercheurs traitent la problématique du foulard à l'école, au collège et au lycée. Lorsque nous avons commencé à réfléchir sur l'objet de notre étude, notre choix s'est immédiatement porté sur l'université, car aucun problème relatif au port du foulard n'y avait été signalé par les médias. Nous nous sommes demandé alors si cette pseudo quiétude et la liberté d'afficher leur religiosité ne cachaient pas chez les étudiantes des conflits non visibles. À ce sujet, Venel (1999), spécialiste en science politique, est la seule à avoir exploré le lien entre le rapport à la religion des étudiantes françaises voilées et leurs stratégies identitaires en interrogeant leurs représentations. Pour notre recherche doctorale, nous nous sommes intéressées à l'identité religieuse de ces jeunes femmes tout en l'articulant à leur rapport aux savoirs. En effet, nous avons constaté, dans notre enquête exploratoire, deux formes de conflits des savoirs dans le discours de trois jeunes femmes interviewées: des conflits interpersonnels (institutionnels) confrontant l'étudiante musulmane portant le foulard à l'institution laïque, le plus souvent représentée par un professeur d'université, et des conflits internes vécus par la jeune femme. Nous avons alors supposé que ces conflits étaient dus à la coexistence, voire à l'antagonisme, de savoirs de nature différente : laïque et religieuse.

Nous avons construit la problématique des conflits de savoirs en tenant compte des rapports de domination sociopolitique des savoirs et du clivage majoritaire/minoritaire directement lié à celui de dominant/dominé. Nous avons par ailleurs, introduit un volet psychanalytique qui étaye un aspect de l'analyse clinique du discours des étudiantes moutahajibâte ${ }^{8}$ et qui interprète le processus de voilement à partir de la théorie du psychanalyste Anzieu sur le « Moi-peau ».

Les savoirs nouveaux de la socialisation secondaire acquis par l'individu, peuvent provoquer un problème «de consistance entre les intériorisations originales et nouvelles » (Dubar, 1996, p. 100) difficile à résoudre. C'est le cas lorsque l'acquisition des nouveaux savoirs n'est pas conforme aux savoirs construits antérieurement et que la réalité sociale (subjective), construite lors de la socialisation primaire, se transforme radicalement. C'est alors que la socialisation secondaire peut «constituer une rupture par rapport à la socialisation primaire » (Idem). Dans le cas des étudiantes de notre enquête,

\footnotetext{
${ }^{7}$ Nous avons établi une typologie de la signification du foulard islamique porté par de jeunes musulmanes françaises sur la base de travaux menés par F. Khosrokhovar sur « l'islam des jeunes » en France, par l'anthropologue N. B. Weibel sur les femmes voilées en France et en Allemagne, par la politologue N. Venel, sur les étudiantes musulmanes voilées à l'université, et F. Zouari sur l'actualité du foulard en France. Pour plus de précisions, voire Triki-Yamani, A. (2004). Conflits internes des savoirs chez les étudiantes musulmanes portant le foulard islamique. Carrefour de l'éducation, ss la dir. de Mosconi N., janvier-juin, n¹7, pp. 25-26. ${ }_{8}^{8}$ « Moutahajibâte » pluriel de « moutahajiba », de la racine et du verbe hajaba, qui signifie cacher.
} 
cette rupture ${ }^{9}$ se produit lorsqu'elles choisissent d'opter pour un islam radical car elles en viennent à reconnaître que le monde représenté par leurs parents, ce même monde qu'elles ont précédemment considéré comme pré-donné, est un monde dont elles ne veulent plus, puisque trop traditionnel et ne correspondant plus à leur idéologie. La rupture est nécessaire car l'infériorité intériorisée par ces jeunes femmes, pendant leur socialisation primaire, face à l'Autre, le Français, n'appartient désormais qu'aux classes inférieures auxquelles s'identifient les parents. En réalité, cette volonté de séparation d'avec la communauté de leurs parents est très complexe car ces jeunes femmes musulmanes ont tendance à être assimilées aux immigrants que sont leurs parents, alors qu'elles sont nées en France. Par ailleurs, le système de classification des savoirs dans la société obéit à celui de la catégorisation sociale. Celui qui détient le statut sociopolitique le plus hiérarchisé, possède automatiquement le savoir qui fait autorité. Les savoirs sont ainsi reliés aux statuts sociaux et deviennent même "des outils importants de distinction sociale: en témoigne le rôle qu'ils jouent dans l'exercice du pouvoir et de l'autorité » (Hatchuel, 2005, p.29).

C'est par rapport à la normalité de la société française, le «Français de souche », en matière d'identité sociale, que les étudiantes voilées représentent une particularité stigmatisée. En réalité, elles sont affublées de deux stigmates : le premier est un stigmate visible, religieux, dont le symbole est le foulard islamique; le second, est un stigmate moins visible, et même parfois complètement invisible chez certaines, hérité des parents : l'origine maghrébine. Pour essayer de comprendre le rejet du stigmate hérité des parents, il faut remonter au contexte historique et juridique de la colonisation du Maghreb par la France. En effet, l'image coloniale de «l'indigène» a laissé place à celle postcoloniale de «l'arabe » qui, dans les années 60, représente en France le plus souvent le travailleur immigré algérien dont le statut social reste toujours infériorisé au point qu'il constitue "la catégorie la plus basse du classement social » (Lorcerie, 2003, p. 82). C'est de ce stigmate dévalorisant du père que les étudiantes musulmanes veulent se débarrasser, à la fois, en réussissant à l'école et à l'université et en adoptant le hijâb distinct de l'habit de la mère, qu'il soit traditionnel ou occidental.

Pour problématiser le processus de couvrement des étudiantes moutahajibâte, nous nous proposons d'adopter trois approches théoriques abordées dans différentes recherches concernant les femmes musulmanes portant le hijâb (Weibel, 2000), ou plus largement, les jeunes femmes issues de l'immigration maghrébine (Lorcerie, 2003). La première approche,

\footnotetext{
${ }^{9}$ Pour plus de détails, voir chapitre sur « l'acquisition des savoirs chez les étudiantes portant le foulard islamique » (Triki-Yamani, 2004, pp.30-34)
} 
psychosociologique, expliquera les stratégies identitaires adoptées par les jeunes femmes en réaction à la stigmatisation dont elles font l'objet (Goffman, 1975). La seconde, ethnologique, étudiera l'acte de porter le foulard comme un rite de passage (Weibel, 2000). Enfin la dernière approche, psychanalytique, relative au concept du «Moi-peau» d'Anzieu (1995), prendra en compte la fonction de protection du foulard.

Nous avons interviewé dix-sept étudiantes françaises moutahajibâte d'origine maghrébine poursuivant leurs études supérieures dans deux universités, parisienne et de la région parisienne. Les entretiens non-directifs se sont déroulés entre 2000 et 2002, après la diffusion de la circulaire Bayrou (1994) ${ }^{10}$ et avant la promulgation de la loi sur l'interdiction des signes religieux à l'école publique $(2004)^{11}$. Toutes fréquentent deux lieux de diffusion du savoir, l'université et la mosquée, et détiennent deux formes de savoirs: les savoirs scolaires, universitaires, et les savoirs islamiques allant de la simple connaissance du Coran et de la Sunna du Prophète en français à des savoirs religieux plus complexes, dans le cadre d'un apprentissage plus spécialisé relevant des programmes des sciences islamiques. Les jeunes femmes appartiennent à des familles dont les pères sont ouvriers ou ouvriers qualifiés. Trois étudiantes (deux du premier groupe et une du second) ont des parents détenant des diplômes universitaires.

Une première analyse thématique des entrevues nous a éclairées sur le lien entre la stratégie identitaire et le rapport aux savoirs des jeunes femmes. Dans la seconde, nous avons analysé leur discours à l'aide de deux approches, compréhensive et clinique, afin de comprendre ces conflits et leurs modes de gestion. Ainsi, dans la première partie de l'article, nous nous intéressons de plus près aux logiques et stratégies identitaires mises en place par les étudiantes moutahajibâte de notre enquête. Nous y explorons plus particulièrement le lien entre la correction de leur(s) stigmate(s) et leur rapport conflictuel aux savoirs. La seconde partie traite l'analyse des conflits des savoirs chez les étudiantes moutahajibâte en interrogeant la manière dont elles se représentent leur rapport (conflictuel) aux savoirs.

\footnotetext{
${ }^{10}$ La circulaire Bayrou diffusée dans les établissements scolaires à la rentrée 1994 était censée aider les chefs d'établissement à prendre la décision d'exclure les élèves ne respectant pas le règlement intérieur de l'école. Elle stipulait, entre autres, qu'il n'était « pas possible d'accepter à l'école la présence et la multiplication de signes si ostentatoires que leur signification est précisément de séparer certains élèves des règles de vie communes de l'école » in Circulaire n¹649 du 20 septembre 1994, in B.O., n³5, 29 septembre 1994.

${ }^{11}$ En mars 2004, la promulgation de la loi interdisant les signes religieux ostensibles à l'école publique vient mettre fin au port du foulard islamique qui est le seul vêtement qui pose vraiment problème.
} 


\section{RAPPORT AUX SAVOIRS ET STRATÉGIES IDENTITAIRES DES MOUTAHAJIBÂTE}

L'analyse thématique nous a permis de définir les caractéristiques de deux groupes constitués de sujets issus de familles pratiquant deux types différents d'islam. Le premier groupe est constitué de cinq jeunes femmes ayant très tôt porté le voile et baigné dans un "islam-action» défini par Weibel comme "l'engagement dynamique au service de la cause religieuse ou l'engagement individuel face à Dieu dans lequel la dimension politique peut être absente. » (2000, p.62). Le second comprend les douze autres étudiantes dont les familles pratiquent un islam-tradition, islam importé par les parents ou grands-parents du pays d'origine, pratiqué par la majorité des musulmans de France et basé sur les pratiques religieuses et traditionnelles consistant principalement à respecter le jeûne, à célébrer les fêtes musulmanes et à accomplir la prière (plutôt par les aînés que par les jeunes d'origine maghrébine). Pour ces sujets du second groupe qui ont revêtu le hijâb une fois à l'université, l'identification au groupe $\mathrm{d}^{\prime}$ " agrégation » ${ }^{12}$ (Weibel, 2000) des femmes musulmanes voilées s'accompagne de l'abandon de l'islam traditionnel des parents pour adopter une forme de religion plus engagée intellectuellement et parfois politiquement: l'islam-action.

Nous avons pu constater que les conflits de savoirs chez les étudiantes moutahajibâte sont liés aux stratégies et logiques identitaires qu'elles mettent en place en réaction à leur stigmatisation. Le foulard islamique est un indicateur visible de l'appartenance des jeunes femmes à un islam-action. D'autres paramètres tels que la fréquentation de la mosquée et la poursuite d'études islamiques dans des instituts spécialisés, en plus de leurs études universitaires, viennent renforcer cette adhésion à l'islam-action.

Dans la construction de leur «nouvelle identité », les dix-sept sujets de notre enquête finissent toutes par adopter la stratégie du « retournement du stigmate » (Goffman, 1975) en revendiquant pour leur particularité religieuse, " un caractère de généralité» (Lorcerie, 2003, p. 36). Cette exigence de reconnaissance par les étudiantes moutahajibâte est détectable, dans leurs discours, par le jeu du « Nous» et du «Eux», le « Nous», faisant allusion au groupe de référence «des frères et sœurs musulmans », et le «Eux», aux représentants de la normativité française, « les Français de souche », ou alors, à la communauté et à la famille maghrébine, dont l'islam n'est plus conforme à celui que pratiquent désormais les jeunes femmes.

La stratégie du retournement du stigmate consistant, selon Goffman, en la combinaison «différenciation/ valorisation», est une stratégie réactive,

\footnotetext{
${ }^{12}$ Voir plus bas, note $\mathrm{n}^{\circ} 11$, sur la définition des trois périodes de la prise du hijâb selon Weibel (2000).
} 
appliquée par les étudiantes moutahajibâte, et mettant en valeur le stigmate visible qu'est le foulard islamique, en en faisant l'emblème de leur singularité et de leur affirmation dans la société française. L'exploitation et l'entretien de leur stigmate « religieux » passe par la valorisation, voire la survalorisation des savoirs religieux sur l'islam par rapport aux savoirs laïques.

La stratégie de l'inversion du stigmate a été précocément adoptée par les étudiantes du premier groupe, qui ont porté le foulard islamique au collège ou au lycée. Ces jeunes filles ne se sont pas contentées à ce moment-là, de la stratégie de $«$ l'excellence $»{ }^{13}$ ou de celle de $~ "$ l'instrumentalisation du stigmate ${ }^{14}$ comme les jeunes femmes du second groupe, qui n'ont porté le hijâb qu'une fois arrivées à l'université. Collégiennes ou lycéennes au moment de l'application de la circulaire Bayrou (septembre 1994), quatre des cinq étudiantes, désirant poursuivre leurs études, renoncent à l'affichage de leur appartenance à un islamaction, pratiqué par leur famille. Durant cette période de renoncement, elles sont confrontées à des conflits internes (Triki-Yamani, 2004) dus à la domination des savoirs laïques scolaires sur leurs savoirs religieux.

Les «affaires du foulard islamique » à l'école française, fortement médiatisées, ont contribué à la vulgarisation du stigmate que portaient en elles certaines collégiennes musulmanes. Avec l'application de la circulaire Bayrou à partir de la rentrée scolaire 1994, les collégiennes et lycéennes persistant à afficher leur stigmate au sein des établissements ont le plus souvent été sanctionnées par l'exclusion. L'une des cinq étudiantes interviewées du premier groupe a subi cette exclusion pendant quelques mois avant d'accepter d'ôter son foulard pour pouvoir intégrer un autre collège. Contraintes d'abandonner leur hijâb ou de le troquer contre un substitut (bandana, bonnet, foulard africain...), lorsque la direction de l'établissement scolaire le permettait, les élèves musulmanes le remettaient une fois sorties de l'enceinte du collège ou du lycée. Elles ont donc persisté à se singulariser en dehors de l'école. Trois autres étudiantes du premier groupe, qui se sont résolues à couvrir leurs cheveux par un tissu qui n'avait pas de connotation religieuse, ont pu continuer à se différencier autrement. Elles tendaient plutôt vers la stratégie de «la différenciation sans dévalorisation de soi » ou stratégie de "l'instrumentalisation du handicap ». La cinquième jeune femme, plus âgée que les autres et déjà à l'université au moment de l'application de la circulaire Bayrou, a quant à elle, appliqué très tôt la stratégie du retournement du stigmate.Arrivées à l'université, les jeunes femmes

\footnotetext{
${ }^{13}$ La stratégie du retournement du stigmate ou stratégie de similarisation/valorisation de soi consiste à rechercher l'excellence en assumant son stigmate : ce qui est possible pour l'Autre (le Français de souche) est possible pour l'individu issu de l'immigration.

${ }^{14}$ Dans le cas de la stratégie de l'instrumentalisation du stigmate ou stratégie de différenciation sans dévalorisation de soi, l'individu assume son stigmate et tend même à l'instrumentaliser.
} 
$\mathrm{du}$ second groupe œuvrent toutes pour la reconnaissance de leur islamité en affichant le hijâb toléré par l'institution. Certaines d'entre elles ont pu adopter des logiques et des stratégies en réaction à des situations conflictuelles vécues, les ayant le plus souvent confrontées à l'institution laïque. C'est le cas d'une des étudiantes qui ne s'est pas encore totalement détachée de la stratégie d'excellence. En effet, lorsque les professeurs lui suggèrent de continuer en maîtrise de maths ou de renoncer au foulard, elle estime qu'elle "ne vaut pas moins que les autres ». Elle déclare avoir étudié et réussi à accéder à l'université comme n'importe quel autre étudiant français dans le même cas qu'elle. Elle trouve alors injuste et injustifié d'être stigmatisée par le seul fait de porter le foulard puisqu'elle a le niveau requis pour préparer le CAPES (Certificat d'aptitude des professeurs de l'enseignement secondaire).

La seule différence entre les deux groupes concerne le cheminement spirituel des jeunes femmes. Nous entendons par cheminement spirituel, l'évolution, la progression de l'étudiante musulmane dans sa réflexion, son questionnement sur la religion musulmane et sur ses piliers ainsi que sur le port $\mathrm{du}$ foulard, prescription coranique. Ce cheminement est étroitement lié à l'acquisition de savoirs religieux intellectualisés par la jeune femme, différents des savoirs inculqués par les parents, qui sont le plus souvent des savoirs traditionnels sur la religion et ses pratiques. L'acquisition de savoirs religieux se fait la plupart du temps de manière autodidacte et par le biais de la fréquentation de la mosquée, lieu privilégié d'apprentissage de ces savoirs. Alors que le cheminement spirituel des sujets du premier groupe est un parcours collectif, qu'il soit familial ou communautaire, celui des jeunes femmes du second groupe est plus singulier, individualisé puisque les étudiantes font un effort personnel d'intellectualisation de l'islam que la plupart des parents ne peuvent suivre, souvent à cause d'un capital scolaire très faible.

Par ailleurs, la stratégie de retournement du stigmate contraint toutes les étudiantes de notre enquête à s'autoexclure du marché de l'emploi. Face à cette exclusion de la vie active, elles envisagent trois formes de conduites.

(1) Certaines d'entre elles résolvent ce problème en choisissant de s'enfermer dans leur communauté religieuse et de se mettre au service de ses membres. Il s'agit en général de jeunes femmes désirant enseigner mais ne pouvant le faire à l'école publique. Elles affirment ainsi leur particularité religieuse dans un cercle communautaire qui les accepte dans leur différence. Leur mission consistera le plus souvent à éduquer les futures générations de musulmans à un islam-action, en créant des écoles et associations privées destinées à accueillir tous les élèves musulmans qui seront en rupture de banc avec l'école publique. 
Leurs savoirs et diplômes universitaires seront ainsi instrumentalisés au profit d'un enseignement empreint d'une idéologie islamique. Cette instrumentalisation des savoirs acquis lors de leur formation universitaire au profit de la diffusion d'un islam-action peut constituer un mode de gestion des conflits internes des savoirs chez les étudiantes moutahajibâte. En effet, les savoirs laïques et universitaires qu'elles détiennent sont exploités dans le but de mieux diffuser un islam intellectuel et scripturaire auprès des élèves musulmans, islam qu'elles ont dû apprendre souvent de manière autodidacte et parfois conflictuelle.

Ces écoles musulmanes privées devront, pour pouvoir fonctionner, se conformer aux programmes en vigueur dans les établissements scolaires publics. En revanche, le danger de cette forme de transmission des savoirs, non contrôlée par les institutions étatiques et dans laquelle les principes de la laïcité sont laissés pour compte, réside dans le type d'idéologie islamique diffusée.

(2) D'autres étudiantes espèrent que les mentalités « évolueront » et qu'elles parviendront un jour à se faire accepter avec leur foulard sur le lieu de leur travail. Dans ce cas, les jeunes femmes ne se remettent jamais en question. Elles ont même tendance à incriminer la société française et les institutions laïques et à se poser en victimes de leur intolérance. Il est à noter que cette stratégie de l'autoexclusion concerne des étudiantes qui n'ont pas de projet professionnel bien défini. Cette stratégie est d'autant plus vouée à l'échec que la fonction publique interdit le port du foulard islamique au sein de plusieurs de ses secteurs (établissements scolaires, cycles primaire et secondaire, hôpitaux, postes)

Quatre étudiantes sur dix-sept envisagent d'exercer leur fonction dans une société musulmane. Trois d'entre elles avancent que la pratique de leur religion est incompatible avec les lois républicaines, et plus particulièrement, avec la laïcité, et projettent de s'expatrier dans un pays où on acceptera qu'elles exercent leurs fonctions couvertes de leur khimâr.

(3) Enfin, seule une étudiante accepte de se défaire de son foulard pour travailler. Le projet de vie des jeunes femmes moutahajibâte est clairement fondé sur une idéologie islamique fondamentaliste. Quatre d'entre elles au moins, envisagent de construire leur avenir, et surtout celui de leurs enfants, dans une société compatible avec leur idéologie religieuse. Les autres resteront en France pour vivre en marge de la société. La plupart des étudiantes ressentent le besoin d'aider leur prochain et de servir plus particulièrement leur communauté religieuse. Que ce soit auprès de leurs enfants ou au sein de la communauté 
maghrébine, la plupart d'entre elles se sentent investies d'une mission, celle de répandre, par n'importe quel moyen, l'islam-action.

Le rapport aux savoirs des étudiantes musulmanes de notre enquête est donc indissociable de leur projet de vie, de leur rapport à l'islam-action. Tout semble s'organiser autour de leur idéologie: leur avenir professionnel, l'éducation de leurs enfants, leur vie restreinte à la communauté religieuse. Les savoirs glanés tout au long de leurs parcours scolaire et universitaire et leurs diplômes universitaires ne serviront que d'instruments pour mieux exploiter et répandre leurs savoirs religieux islamiques. Nous pourrions même croire à un retour au mode de vie des musulmans du temps du Prophète. En témoigne la mission de transmission «du Message » dont se sentent investies certaines des étudiantes, au point de faire référence aux femmes du Prophète qui sont des modèles pour toutes les musulmanes, telles Khadidja, femme d'affaires, qui fut la première à entendre l'appel de l'islam et, Aïcha, fine connaisseuse du Coran et ayant joué un rôle capital dans la transmission des hadiths.

\section{MODES DE GESTION DES CONFLITS DE SAVOIRS CHEZ LES ÉTUDIANTES MOUTAHAJIBÂTE}

Les conflits internes et interpersonnels chez les étudiantes voilées s'expriment à travers ce qu'elles appellent leur « cheminement spirituel». Nous pouvons alors supposer que les conflits internes dus au couvrement sont distincts selon qu'ils concernent les étudiantes ayant porté le foulard islamique pour la première fois au collège ou au lycée, et celles qui l'ont porté pour la première fois à l'université. Concernant le premier groupe, nous pouvons penser que les conflits interpersonnels, ayant opposé les jeunes filles à l'institution scolaire, au collège et au lycée, ont entraîné des conflits internes chez les adolescentes. Quant aux jeunes femmes du second groupe, nous supposons que la résolution des conflits internes par le port du foulard, engendre chez elles des conflits interpersonnels.

\section{Essai d'interprétation psychologique des conflits vécus par les jeunes filles du premier groupe}

Dans le cas des jeunes filles du premier groupe ayant été contraintes de renoncer au port du foulard islamique pour poursuivre leur scolarité, les agressions externes peuvent être générées par l'ultimatum imposé aux collégiennes et lycéennes, à savoir celui de retirer le hijâb. Ces agressions peuvent être stoppées à une seule condition, celle d'abandonner le collège ou le lycée et d'opter, par exemple, pour des études par correspondance, choix fait par de nombreuses 
élèves de confession musulmane, surtout après l'application de la loi pour la Laïcité (2004).

Si cette option est écartée, les agressions externes vécues par les collégiennes et lycéennes sont causées par l'obligation de se plier à un pouvoir institutionnel qu'elles vivent comme arbitraire et leur imposant de mettre en cause leur fidélité à une croyance religieuse étrangère à ce pouvoir. À ce conflit interpersonnel s'ajoute un conflit plus personnel engendré par des agressions internes dues à la cohabitation de deux formes de savoirs savants chez les jeunes filles : les savoirs scolaires et les savoirs religieux. Ce conflit interne est par conséquent plus particulièrement généré par l'attachement des étudiantes du premier groupe à la fois aux savoirs de la religion musulmane et aux savoirs scolaires laïques.

Pour gérer leur rapport conflictuel à l'institution scolaire, les jeunes filles sont forcées de renoncer aux savoirs religieux, qui, selon elles, intiment à la femme musulmane de porter le foulard islamique. Dans ce cas, le conflit interpersonnel est géré, mais en apparence seulement, puisque les jeunes filles finissent par se présenter tête nue (ou, dans le meilleur des cas, couvertes d'un tissu sans connotation religieuse, un bandana, un béret...) dans les établissements scolaires, alors qu'une longue période de conflits internes se dessine pour elles. Elles ont tendance, en évoquant cette période douloureuse de leur scolarité, à citer dans leur discours des vocables à connotation forte tels que "honte ", « humiliation », « souffrance », " nudité »... Ces sentiments négatifs sont des affects déplaisants engendrés par les conflits interpersonnels vécus par les jeunes filles face à l'institution scolaire. Ces affects constituent dans le même temps des signaux de la défense psychique mise en place par les collégiennes. Si l'on se réfère à la théorie de la défense selon $\mathrm{S}$. Freud, le conflit interne devient l'enjeu d'une opposition qui se joue "entre les excitations externes qu'on peut fuir, grâce au pare-excitations, et les excitations internes » (Laplanche, Pontalis, p. 108).

Ces conflits internes vécus par les étudiantes tout au long de leur scolarité, et avant d'accéder à l'université où elles sont libres de se vêtir comme elles le souhaitent, sont plus particulièrement occasionnés par la domination du pouvoir institutionnel laïque, dispensateur de savoirs laïques, sur l'institution religieuse du groupe minoritaire qui transmet les savoirs savants religieux. Ces conflits renforcent la position sociale d'infériorisation des jeunes femmes longtemps vécue par le biais de celle de leurs parents. Alors qu'elles pensaient s'en débarrasser par l'acquisition des savoirs scolaires dominants, elles sont contraintes de subir les savoirs scolaires au détriment des savoirs religieux au lieu de les utiliser comme arme pour une meilleure promotion et émancipation 
sociales. Nous pouvons donc avancer que les conflits internes ne disparaissent qu'une fois que le port du foulard est à nouveau possible, et compatible avec l'acquisition des savoirs savants profanes pour les jeunes femmes du premier groupe.

Une fois arrivées à l'université, ces mêmes étudiantes ne sont plus contraintes de subir les savoirs universitaires au détriment des savoirs religieux. $\mathrm{Au}$ contraire, certaines instrumentalisent les savoirs universitaires, lä̈ques, au profit des savoirs sacrés (Triki-Yamani, 2004, pp. 31-33). Cette instrumentalisation peut être considérée, dans certains cas, comme un mode de gestion des conflits internes des savoirs chez les étudiantes voilées. L'université, en tant que terrain neutre, permet ainsi un rapport au savoir libre et sans risque. Savoir, pour l'étudiante, est en relation avec liberté d'accès au savoir. Par ailleurs, l'esprit critique revendiqué par les sciences rationnelles, et inculqué par l'école française permet aux jeunes femmes de ne pas accepter certains savoirs, de source religieuse, critiquables et s'éloignant de la vérité divine. Cependant, ces dernières - et c'est également le cas pour celles du second groupe - ne distinguent pas la sphère publique du savoir de celle privée du croire. Ainsi, le port du hijâb à l'université constitue à la fois une manifestation publique du croire et un affermissement du croire dans l'apprentissage des savoirs religieux et profanes. (Le rapport au(x) savoir(s) des étudiantes n'est pas séparé de leur rapport au religieux. Le fait même que l'instruction soit l'une des prescriptions premières révélées par le texte sacré est la preuve que le savoir est indissociable du religieux. Le savoir fait partie de la vie spirituelle, religieuse, puisque c'est une obligation pour tout musulman. Les conflits provoqués par les deux formes de savoirs ne sont que le reflet d'une lutte continue pour sauvegarder les savoirs islamiques, dominés par des savoirs jugés trop rationnels, et souvent profanes, légitimés par une société sécularisée qui n'admet pas le sacré et le religieux dans le champ intellectuel et scientifique.

Le regard que porte le professeur d'université sur l'étudiante moutahajiba ne se limite pas uniquement à l'animosité que provoque le port du foulard, mais il s'étend au conflit engendré par l'éventuelle détention par la jeune femme de savoirs religieux systématiquement discrédités et refusés par le représentant de l'institution laïque ${ }^{15}$. Ce qui accentue le conflit interpersonnel entre l'étudiante voilée et le professeur d'université, c'est le type de rapport aux savoirs religieux que possèdent l'un et l'autre. En effet, l'étudiante musulmane

\footnotetext{
${ }^{15}$ Sur ces conflits interpersonnels entre étudiantes et professeur, nous nous référons, entre autres, au témoignage de M. sur une situation conflictuelle vécue avec son professeur d'histoire à l'université concernant « le voyage nocturne » du Prophète. Ce voyage est un « voyage imaginaire » pour le professeur et il est impossible pour lui qu'il se soit produit d'un point de vue historique. Pour la jeune femme, cet événement contenu dans la Révélation ne peut être que vrai.
} 
qui se fait le porte-parole de l'institution religieuse musulmane, détient un rapport non sécularisé aux savoirs religieux, alors que le professeur représentant de l'institution laïque, manifeste un rapport sécularisé aux savoirs religieux. Par ailleurs, nous ne pouvons occulter la domination des savoirs rationnels détenus par le professeur sur les savoirs des étudiantes moutahajibâte. Légitimés politiquement et socialement, les savoirs savants et rationnels sur les religions détenus par les représentants de l'institution laïque, sont reconnus comme fondés, alors que ceux de l'étudiante musulmane voilée restent critiquables du fait de leur statut non institutionnalisé et rejeté par l'institution laïque.

\section{Essai d'interprétation des conflits internes et interpersonnels des savoirs chez les étudiantes du second groupe}

Ce que nous pouvons déduire de l'analyse des témoignages des étudiantes du second groupe, c'est que chacune des périodes qu'elles ont vécues lors du processus de prise du hijâb, engendre des conflits psychiques mais de nature différente selon la période. Pendant la période d'attente ${ }^{16}$, nous avons constaté chez tous les sujets des conflits internes psychiques. Toutes les étudiantes témoignent d'un manque qu'elles relient à un sentiment d'inachevé dans leur parcours spirituel. Ces conflits internes sont résolus, pour ces mêmes jeunes femmes, au moment de la prise du hijâb. En outre, les nombreux membres constituant la communauté des «frères et sœurs musulmans » leur donnent, à elles qui sont nouvellement moutahajibâte "une impression de puissance, car elle(s) possède(ent) la force de la quantité, de l'espace et du temps. » (Chiland, 1983, p.68). Cependant, ces conflits laissent place à de nouveaux conflits d'ordre interpersonnel engendrés par l'adoption du foulard. Ces derniers sont le plus souvent provoqués par des agents extérieurs et agresseurs tels que le regard malveillant, réprobateur, inquisiteur voire rejetant de l'autre.

Nous constatons, tout d'abord, que la période d'attente se caractérise, chez les jeunes femmes du second groupe par l'identification d'un sentiment de manque alors que deux piliers de la religion, le ramadan et la prière, sont d'ores et déjà respectés. Ce manque est dû à l'absence du foulard pendant la période d'attente, qui se caractérise ainsi par un déséquilibre chez les jeunes femmes du second groupe. Le ramadan et la prière font partie de l'éducation religieuse transmise par les parents à leurs enfants. En effet, l'observance de ces deux piliers par une grande majorité des membres de la communauté maghrébine est un

\footnotetext{
${ }^{16}$ La période d'attente se situe avant la période de séparation et se caractérise par une volonté de recherche plus approfondie de la religion. La période de séparation marque la limite entre l'avant et l'après foulard. La dernière étape du processus de prise du hidjab est celle d'agrégation se caractérisant par l'admission de la jeune femme dans le cercle des musulmanes pratiquantes et plus particulièrement, au sein de la communauté religieuse des « frères et sœurs musulmans »
} 
moyen de renforcer leur identité puisque, dans leur société d'origine, la religion est une composante quasi présente dans le quotidien des Maghrébins. Les deux pratiques religieuses constituent pour les jeunes femmes du second groupe une injonction religieuse externe issue de la communauté musulmane dans son ensemble. En revanche, le port du foulard islamique est un acte plus personnel et réfléchi qui complète le cheminement spirituel des étudiantes. Cette démarche, rarement suggérée par les parents des sujets du second groupe, voire parfois critiquée par ces derniers, est une manière de se démarquer d'un islam devenu trop traditionnel et désuet, représentant dorénavant la génération ayant immigré depuis plusieurs décennies en France. Pour s'en démarquer, les jeunes femmes entreprennent une action personnelle en s'engageant dans une relation plus intimiste avec Dieu. C'est comme si elles excluaient tout intermédiaire « humain » entre elles et le Créateur. Cependant, un obstacle subsiste entre elles et Dieu. En effet, le port du foulard islamique par les femmes marque le respect d'une certaine distance face à Dieu, sans laquelle une offense lui serait faite.

Le second thème récurrent concerne le déclic décrit par les étudiantes, point de départ de la prise de conscience de la nécessité du voilement pour parvenir à un niveau supérieur de la foi. Nous avons pu constater que l'élément déclencheur dans la prise du hijâb est souvent lié à des événements sacrés, comme l'approche du mois du ramadan et les prières effectuées collectivement à la mosquée, ou alors, à la prescription coranique et aux preuves de la Sunna relatives à la tenue vestimentaire de la femme musulmane. Nous remarquons ici l'importance du référent religieux dans le passage à l'acte.

Les étudiantes voilées passent d'un islam-culture trop désuet à un islamaction, intellectualisé. La démarche personnelle d'intellectualisation de l'islam propre à la période d'attente de la prise du hijâb est un passage obligé pour la jeune femme qui tente de rétablir le déséquilibre psychique. Au bout de cette réflexion, l'étudiante finit par décider de porter définitivement le foulard islamique. Elle se débarrasse également des savoirs traditionnels des parents sur la religion, et par la même occasion du statut de dominé du père, ouvrier immigré, appartenant à la première génération et resté attaché aux traditions ancestrales de son pays et de son groupe social d'origine.

$\mathrm{Au}$ moment de la remise en question des jeunes femmes, autrement dit pendant la période d'attente, le Moi-peau ${ }^{17}$ n'assure plus ses fonctions

\footnotetext{
${ }^{17}$ Notre réflexion sur les conflits internes des savoirs, vécus par l'étudiante portant le foulard, s'appuie sur le concept du Moi-peau développé depuis plus de dix ans par D. Anzieu. Nous avons fait l'hypothèse que ce concept pouvait, par analogie, s'appliquer au foulard, en le considérant comme une " seconde peau » ou comme une enveloppe de renforcement du Moi-peau (Triki-Yamani, 2004, p. 36). Par ailleurs, D. Anzieu donne une définition complète du Moi-peau dans la première édition du « Moi-peau » (1985) : «Le Moi-peau sert à
} 
essentielles de contenance et de protection. Le conflit interne chez les étudiantes musulmanes sur le point d'adopter le foulard islamique, est, dans un premier temps, généré par l'opposition entre des agressions externes de deux types : les premières agressions exogènes proviennent du regard, voire du jugement de la communauté des frères et sœurs musulmans partisans de l'islam-action et pour qui le port du foulard est une injonction à laquelle il est impossible de se soustraire, et les secondes sont symbolisées par le regard désapprobateur des parents appartenant à l'islam-culture, craignant pour l'avenir socio-professionnel de leurs filles. Les étudiantes musulmanes savent d'ores et déjà que ce regard critique s'étendra aux autres personnes, celles de la rue, leurs professeurs d'université, les étudiants, le personnel administratif... Le regard de l'autre sur les jeunes femmes en passe de prendre le foulard est donc multiple et contradictoire.

Cependant, le conflit interne ne fait pas uniquement l'objet de l'opposition entre des agents agresseurs exogènes de types différents. En effet, il faut compter également avec le regard de l'Autre, omniprésent et tout-puissant, celui de Dieu. L'injonction interne, liée à la relation intimiste de la jeune femme à Dieu, atteint son paroxysme à un moment donné de la période d'attente et prend le dessus sur les agents agresseurs externes. C'est ce que les jeunes femmes nomment «le déclic », à savoir, le moment opportun et irréversible de la prise du hijâb.

Le conflit interne est ainsi réglé par la prise du foulard. Le foulard devient ainsi une enveloppe textile de renforcement qui remplit, à l'instar du Moi-peau, les fonctions fondamentales de la peau.

La première fonction est celle de contenance; le foulard contient les cheveux et le crâne. L'enveloppe textile que constitue le foulard recouvre l'enveloppe corporelle, la peau, à l'instar de n'importe quel autre vêtement. Nous croyons que cette « seconde peau », qui est en réalité une enveloppe textile, vient renforcer l'enveloppe psychique de la jeune femme.

Le foulard contient réellement des parties du corps car il ramasse les cheveux de la même manière que l'épiderme, couche externe de la peau, contient les parties internes du corps (...) Il renforce l'enveloppe psychique (de contenance), cette fois d'un point de vue métaphorique, à des moments cruciaux et conflictuels de la vie de la jeune femme (la période d'attente de la prise du hijâb), qui cherche inconsciemment, à travers le port du foulard, à rétablir la constance (assurée par

l'enfant à se représenter lui-même comme Moi « contenant les contenus psychiques » à partir de l'expérience du corps » (Anzieu, 1995, p. 2) 
la fonction de filtre imposant la limite entre le dedans et le dehors) de son appareil psychique. Pour reprendre les termes de D. Anzieu, le foulard remplit, au même titre que le Moipeau, la fonction d'enveloppe contenante et unifiante du Soi. (Triki-Yamani, 2004, p.37)

La seconde fonction est celle de protection et de filtrage ; le foulard filtre les agressions extérieures. Il protège plus particulièrement des regards malveillants. La fonction de protection est directement liée au registre socio-religieux lorsqu'il s'agit de décrire les symboles du foulard islamique. Le foulard protège du regard concupiscent de l'homme musulman, auquel il est recommandé, dans le verset 30 de la sourate "La Lumière ${ }^{18}$, de baisser les yeux devant une femme étrangère à son foyer. Au cas où cette prescription ne serait pas respectée par l'homme, le verset 31 de cette même sourate ${ }^{19}$ intime à la femme de rabattre son fichu sur sa poitrine afin de ne pas attirer le regard de l'homme. De plus, le verset 59 de la sourate «Les Coalisés ${ }^{20}$ recommande aux femmes musulmanes de se couvrir pour se protéger des regards mal intentionnés des hommes ne respectant pas la prescription du verset 30 de la sourate «La Lumière ».

Le foulard, par sa fonction protectrice, aide ainsi la femme musulmane à préserver sa pudeur, sa chasteté, sa dignité et l'honneur de la famille et de la communauté.

Comment le foulard engendre-t-il des conflits interpersonnels chez les étudiantes du second groupe dans la période de séparation?

Nous avons pu remarquer que la résolution des conflits internes par le voilement était systématiquement suivie de l'apparition de conflits interpersonnels chez les étudiantes voilées.

Si nous entendons par filtrage l'action de filtrer les agressions exogènes au sens que lui donne D. Anzieu, alors le foulard peut remplir cette fonction. Le « regard de l'autre » - mais il ne s'agit plus ici de l'homme, mais de l' «autre », membre du groupe dominant - est considéré comme une agression externe pour la jeune femme dont le foulard engendre souvent l'animosité et la suspicion. (TrikiYamani, 2004). Nous avons pu constater dans l'analyse des entretiens que le regard de l'autre, en tant qu'agent agresseur, peut être multiple. Ce peut être celui

\footnotetext{
${ }^{18}$ «...Dis aux croyants de baisser leurs yeux et de contenir leur sexe : ce sera de leur part plus net. Dieu est de leurs pratiques Informé », in (Berque, 1995, p.375).

${ }^{19} «$... Dis aux croyantes de baisser leurs yeux et de contenir leur sexe, (...) de rabattre leur fichu sur les échancrures de leur vêtement... », idem.

${ }_{20}$ « Prophète, dis à tes épouses, à tes filles, aux femmes des croyants de revêtir leurs mantes : sûr moyen d'être reconnues (pour des dames) et d'échapper à toute offense. Dieu est Tout indulgence, Miséricordieux. », in (Berque, 1995, p.454).
} 
de l'individu croisant la jeune femme portant le foulard dans les lieux publics, celui des deux couples dévisageant $\mathrm{S}$. alors qu'elle est au restaurant en compagnie de son mari, celui du personnel administratif ou dirigeant du département universitaire qui sanctionne $\mathrm{N}$. ou celui du professeure d'université qui montre son désaccord total pour la tenue vestimentaire d'A., alors qu'elle distribue des tracts à l'entrée de l'université.

Même si une pseudo-stabilité psychique est assurée chez les sujets du second groupe lors de la période d'agrégation, les conflits des savoirs ne disparaissent jamais de manière définitive. Ce sentiment de sécurité est généré par l'adhésion des jeunes femmes à l'endogroupe religieux qui leur permet d'affirmer leur particularité religieuse avec plus de force.

\section{CONCLUSION}

Pour toutes les étudiantes, la décision de porter le foulard à l'université constitue l'aboutissement d'une trajectoire réflexive et critique, collective ou individuelle, d'un islam traditionnel réduit à quelques pratiques culturelles. C'est aussi l'acte initiateur d'un processus d'approfondissement des savoirs religieux indissociable d'une prise de risque assumée de confrontation au regard et à la parole des « autres ». Même si les savoirs de la religion sont pour elles consubstantiels à la pratique de leur religion, la fonction critique des savoirs universitaires leur est nécessaire pour un accès libéré aux savoirs religieux et à leur transmission aux futures générations.

La partie empirique de notre recherche nous a permis d'établir un lien non négligeable entre l'identité islamique des étudiantes françaises musulmanes et leur rapport aux savoirs. Le cheminement spirituel, comprenant des rites de passage dont la prise du hijâb est au centre de la construction réactive de l'identité islamique des jeunes femmes, renforce leur rapport à la religion qui se veut être un rapport à l'islam-action. Or cette nouvelle forme d'islam prônée par les musulmans français est un islam scripturaire, faisant référence aux textes fondateurs que sont le Coran et la Sunna. Les hadiths contenus dans la Sunna codifient dans les moindres détails ce qu'il est licite ou illicite de faire dans plusieurs domaines de la vie du musulman : la manière de manger, de se vêtir (très précise aussi bien pour la femme que pour l'homme), de vivre sa sexualité...

Mais l'islam-action ne se limite pas aux comportements individuels de tout musulman. Il agit également sur la sphère communautaire élargie et détermine un véritable projet de société où tout est codifié de manière à imposer un nouveau système de normalité dont le port du foulard par la femme, est l'une 
des composantes «ostensibles » donnant un caractère fondamentaliste, voire extrémiste, à l'islam en général.

Le rapport à l'islam-action des étudiantes musulmanes devient indissociable de leur rapport aux savoirs. Ainsi, les savoirs savants islamiques prennent le dessus sur les savoirs laïques qui ne servent plus que de «passeport» dans une société sécularisée, en marge de laquelle elles ont choisi de vivre. L'islam-action gère ainsi le système des savoirs en occultant le contexte social dans lequel vivent les jeunes femmes. L'institution scolaire et laïque longtemps dominante est supplantée par l'institution religieuse.

L'analyse thématique des entretiens non-directifs nous a permis de distinguer le groupe d'appartenance des sujets de leur groupe de référence. Le passage de l'un à l'autre s'est accompagné de l'abandon de l'islam traditionnel des parents pour adopter une forme de religion plus engagée. Tout dans la vie de ces femmes s'organise autour de cet islam intellectualisé, proche des textes fondateurs.

Nous avons pu déterminer, par ailleurs, que leur rapport à l'islam-action influence leur rapport aux savoirs. Les conflits internes et interpersonnels sont ainsi dus à la survalorisation des savoirs issus de l'islam-action, au détriment des autres savoirs. Les jeunes femmes refusent la reproduction de la domination des savoirs laïques, qui sont l'apanage des représentants de la majorité intellectuelle dominante ; cette même domination subie par leurs parents et contre laquelle elles tentent de se défendre en instrumentalisant l'outil de la domination, les savoirs laïques, pour mieux exploiter et répandre l'islam-action.

Par ailleurs, nous avons constaté, à travers les témoignages des étudiantes voilées que les fonctions psychiques du foulard islamique, rappelant celles du Moi-peau, permettent, à la fois, de contenir la tête, les cheveux et le cou, comme le recommandent d'ailleurs les textes fondateurs (la Sunna est plus explicite à ce sujet que le Coran), et de protéger l'appareil psychique des agents agresseurs internes (opposition entre les deux formes de savoirs) et externes (le regard de l'autre), par l'opération de filtrage.

Nous sommes conscientes que les résultats de notre recherche ne peuvent en aucun cas être généralisables à toutes les étudiantes musulmanes de France, d'origine maghrébine, portant le foulard islamique. Nous avons vu que les conflits internes pouvaient être très singuliers et propres au cheminement religieux et intellectuel des étudiantes. En revanche, la récurrence des thèmes concernant les conflits interpersonnels nous a montré que les jeunes femmes vivaient les mêmes types de situations conflictuelles qui les opposaient systématiquement à l'institution laïque. Toutefois, cette récurrence évidente ne nous permet toujours pas d'étendre cette thèse aux autres étudiantes présentant les mêmes caractéristiques. 
Pour conclure, cette recherche ne prétend pas présumer du rapport aux savoirs des futures générations de « sœurs musulmanes » de France. L'on peut se demander si, avec le temps, ces générations n'abandonneront pas d'elles-mêmes l'islam-action de leurs aînées, lesquelles avaient renoncé à l'islam-tradition de leurs propres parents.

Vingt années se sont écoulées depuis la première «affaire du foulard islamique » à l'école française, période marquée par trois dates importantes : la promulgation de la circulaire Jospin (décembre 1989), celle de la circulaire Bayrou (septembre 1994) et la loi concernant le port de signes religieux à l'école (mars 2004). Or, en 2009, ce n'est plus le hijâb des musulmanes françaises qui suscite la mobilisation médiatique et politique en France mais l'affichage de la burqa par certaines d'entre elles. Le mode de vie des parents adeptes de cet islam-action pourrait se perpétuer à travers les générations montantes à moins que l'État français ne s'implique davantage dans l'intégration des musulmans de France, en tenant compte de leurs particularités culturelles et religieuses.

\section{Bibliographie}

Al-Ashmawy, M.S. (1989). L'islam politique. Alger, Laphomic/ Bouchène.

Anzieu, D. (1995). Le Moi-peau. Paris, Dunod.

Berque, J. (1995). Le Coran, Essai de traduction, Édition revue et corrigée. Paris, Édition Sinbad.

Chiland, C. (1983), L'entretien clinique. Paris, PUF.

Dubar, C. (1996). La socialisation, construction des identités sociales et professionnelles. Paris, Armand Colin.

Gaspard, F., Khosrokhovar, F. (1995). Le foulard et la République. Paris, La Découverte.

Goffman, E. (1975). Stigmate, les usages sociaux du handicap. Paris, Éditions de Minuit.

Hatchuel, F. (2005). Savoir, apprendre, transmettre. Une approche psychanalytique du rapport au savoir. Paris, La Découverte.

Khorokhovar, F. (1997). L'Islam des jeunes. Paris, Flammarion.

Khorokhovar, F. (1995-1996). L’identité voilée. Confluences Méditerranée, n¹6, hiver.

Laplanche, J., Pontalis, J. B. (2002). Vocabulaire de la psychanalyse. Paris, PUF.

Lorcerie, F. (2003). L'école et le défi ethnique, Éducation et intégration. Paris, ESF Éditeur.

Lorcerie, F., Morel, S., (2003). Une politique introuvable. La scolarisation des enfants d'immigrés. In, Lorcerie, F. L'école et le défi ethnique, Éducation et intégration, Paris, ESF éditeur.

Triki-Yamani, A. (2004). Conflits internes des savoirs chez les étudiantes musulmanes portant le foulard islamique. Carrefour de l'éducation, in Mosconi N., janvier-juin, n 17 , pp. 22-42.

Venel, N. (1999). Musulmanes françaises. Des pratiquantes voilées à l'université. Paris, L'Harmattan.

Weibel, B.W. (2000). Par delà le voile, femmes d'islam en Europe. Paris, Éditions Complexe.

Zouari F. (2002). Le voile islamique, Histoire et actualité, du Coran à l'affaire du foulard. Lausanne, Éditions Favre SA.

Amina Triki-Yamani ( $\mathrm{PhD}$ Université Paris $\mathrm{X}$-Nanterre) travaille comme agente de recherche avec Mme Marie McAndrew, Chaire de recherche du Canada sur l'Education et les rapports ethniques à l'Université de Montréal. Elle s'intéresse à la construction du curriculum et le traitement du monde musulman dans le matériel didactique québécois et ontarien. On peut la contacter à : amina.triki.yamani@umontreal.ca. 